\title{
Peritoneal Dissemination of Hepatocellular Carcinoma Treated with a Combination Therapy of Interferon-alpha-2b and Oral Tegafur/Uracil
}

\author{
Yasuhiro Miyake, Yoshiaki Iwasaki, Hidenori Shiraha, \\ Kohsaku Sakaguchi and Yasushi Shiratori
}

\begin{abstract}
We encountered a case of peritoneal dissemination of hepatocellular carcinoma, successfully treated with a combination therapy of interferon-alpha-2b and oral tegafur/uracil. A 67-year-old Japanese man who underwent a hepatectomy developed peritoneal dissemination. A combination therapy of subcutaneous interferonalpha- $2 \mathrm{~b}$ and intravenous 5-fluorouracil was started. Four weeks later, he felt severe general fatigue and nausea, and intravenous 5-fluorouracil was replaced with oral tegafur/uracil. At 3 months after the initiation of chemotherapy, enhanced computed tomography showed markedly reduced peritoneal dissemination. A combination therapy of interferon-alpha- $2 b$ and oral tegafur/uracil is facile and may be effective for extrahepatic metastasis of hepatocellular carcinoma.
\end{abstract}

Key words: systemic chemotherapy, extrahepatic metastasis, 5-fluorouracil

(DOI: 10.2169/internalmedicine.46.6362)

\section{Introduction}

Worldwide, primary liver cancer is the fifth most common cause of cancer-related death with more than 500,000 new cases per year $(1,2)$. During the last 2 decades, increases of primary liver cancer incidence rates have been reported from Australia, Central Europe, the United Kingdom, Japan, and North America $(1,2)$.

Curative treatments, such as resection, liver transplantation, and percutaneous ablation, induce complete responses in a high proportion of patients and are expected to improve survival. However, candidates for the curative treatment are limited. According to a recent study in Japan, only $58 \%$ of the newly registered 18,827 patients with hepatocellular carcinoma could receive curative treatment (3)

The prognosis of advanced hepatocellular carcinoma is still unsatisfactory, and extrahepatic metastasis is a major independent prognostic factor for patients with hepatocellular carcinoma $(3,4)$. The efficacy of systemic chemotherapy for extrahepatic metastasis has been unsatisfactory.
In this report, we describe a Japanese man who developed peritoneal dissemination of hepatocellular carcinoma after hepatectomy, in whom marked tumor regression was found with a combination therapy of interferon-alpha- $2 b$ and oral tegafur/uracil.

\section{Case Report}

A 67-year-old Japanese man was admitted to Okayama University Hospital with hepatocellular carcinoma in February 2005. Hepatitis C virus antibody was positive, while hepatitis B surface antigen was negative. Serum levels of alpha-fetoprotein and des-gamma-carboxy prothrombin were markedly elevated (Table 1). Enhanced computed tomography showed a hepatic mass with early-phase hyperattenuation and late-phase hypoattenuation, measuring $8.5 \times 5.4$ $\mathrm{cm}$ in the posterior segment of the liver (Fig. 1A, B). No ascites was found. In March 2005, a posterior segment resection was performed. Histological examination revealed a moderately differentiated hepatocellular carcinoma arising in cirrhotic hepatic parenchyma (Fig. 1C, D). The postopera-

Department of Gastroenterology \& Hepatology, Okayama University Graduate School of Medicine, Dentistry and Pharmaceutical Sciences, Okayama

Received for publication November 8, 2006; Accepted for publication December 21, 2006

Correspondence to Dr. Yasuhiro Miyake, miyakeyasuhiro@hotmail.com 
Table 1. Laboratory data at the diagnosis of hepatocellular carcinoma and at the diagnosis of peritoneal dissemination

\begin{tabular}{|c|c|c|}
\hline Date & Feburary 2005 & June 20 \\
\hline White blood cell count $\left(/ \mathrm{mm}^{3}\right)$ & 5700 & 3900 \\
\hline Hemoglobin (g/dL) & 15.4 & 9.6 \\
\hline Platelet count $\left(\mathrm{x} 10^{3} / \mathrm{mm}^{3}\right)$ & 281 & 187 \\
\hline Albumin $(\mathrm{g} / \mathrm{dL})$ & 3.7 & 3.1 \\
\hline Total bilirubin (mg/dL) & 1.34 & 1.04 \\
\hline Aspartate aminotransferase (IU/L) & 127 & 95 \\
\hline Alanine aminotransferase (IU/L) & 75 & 47 \\
\hline Alkaline phosphatase (IU/L) & 441 & 490 \\
\hline Creatinine $(\mathrm{mg} / \mathrm{dL})$ & 0.45 & 0.56 \\
\hline Prothrombin time (INR) & 1.07 & 1.14 \\
\hline Hepatitis $\mathrm{C}$ virus $\mathrm{RNA}(\mathrm{KIU} / \mathrm{mL})$ & 4100 & NT \\
\hline Hepatitis $\mathrm{C}$ virus serotype & 2 & NT \\
\hline Alpha-fetoprotein (ng/mL) & 54535 & 45562 \\
\hline Des-gamma-carboxy prothrombin (mAU/mL) & 2576 & 260 \\
\hline
\end{tabular}

NT, not tested.

tive course was uneventful, and his serum levels of alphafetoprotein and des-gamma-carboxy prothrombin decreased after surgery (Fig. 2).

In May 2005, his serum levels of alpha-fetoprotein and des-gamma-carboxy prothrombin increased unexpectedly. Enhanced computed tomography showed no intrahepatic recurrence of hepatocellular carcinoma but peritoneal dissemination (Fig. 3A, B). From July 2005, combination therapy of subcutaneous $6 \times 10^{6}$ units of interferon-alpha- $2 \mathrm{~b}$, three times a week, and intravenous $500 \mathrm{mg} /$ day of 5-fluorouracil, daily for consecutive 5 days a week, was started. Four weeks later, he felt severe general fatigue and nausea. Thus, intravenous 5-fluorouracil was changed to daily oral 300 $\mathrm{mg} /$ day of tegafur/uracil. His general fatigue and nausea disappeared. At 3 months after the initiation of chemotherapy, his serum levels of alpha-fetoprotein and des-gammacarboxy prothrombin decreased again, and enhanced computed tomography showed reduced peritoneal dissemination. Partial response was achieved (Fig. 3C, D).

At 5 months after the initiation of chemotherapy, his serum levels of alpha-fetoprotein and des-gamma-carboxy prothrombin increased again, and intravenous $5 \mathrm{mg} /$ day of cisplatin, daily for 5 days a week, was additionally started. Four weeks later, he felt anorexia again, and intravenous cis- platin was stopped. However, his serum levels of alphafetoprotein and des-gamma-carboxy prothrombin began to decrease. Since then, a combination therapy of interferonalpha- $2 \mathrm{~b}$ and oral tegafur/uracil was continued, and he did not have general fatigue, nausea, jaundice, peripheral edema, or ascites.

At 9 months after the initiation of chemotherapy, he was admitted for swelling of the right leg, fever, and dyspnea. He presented necrotizing cellulitis of the right lower extremity, and administration of antibiotics was started. However, he rapidly developed multiple organ failure and died 4 days later.

\section{Discussion}

Recently, the efficacy of chemotherapy using interferonalpha for advanced hepatocellular carcinoma has been reported $(5,7)$. Especially, in patients with major portal vein tumor thrombosis and without extrahepatic metastasis, the efficacy of a combination therapy of subcutaneous interferon-alpha and intra-arterial 5-fluorouracil is reported to be a $44 \%$ response rate (complete response rate and partial response rate) and $15 \%$ complete response rate (6). However, in patients with extrahepatic metastasis, the efficacy of systemic chemotherapy using interferon-alpha is uncertain. Miyamoto et al (8) reported a case of hepatocellular carcinoma with multiple intrahepatic as well as pulmonary and bone metastases, who was successfully treated by a combination therapy of interferon-alpha and oral tegafur/ uracil. In the present case, peritoneal dissemination of hepatocellular carcinoma without intrahepatic recurrence appeared, and partial response was achieved with a combination therapy of interferon-alpha- $2 b$ and oral tegafur/uracil. Systemic chemotherapy using interferon-alpha may be effective for extrahpatic metastasis.

Several anti-cancer drugs were administered to the present case. The serum levels of both alpha-fetoprotein and desgamma-carboxy prothrombin decreased after the combination therapy of interferon-alpha- $2 b$ and oral tegafur/uracil for four weeks. Furthermore, during the combination therapy of interferon-alpha- $2 b$, oral tegafur/uracil, and intravenous cisplatin, the increasing tendency of both markers decreased. On the other hand, during the combination therapy of interferon-alpha-2b and intravenous 5-fluorouracil, the increasing tendency of serum level of alpha-fetoprotein, but not des-gamma-carboxy prothrombin, decreased. Thus, in the present case, the combination therapy of interferonalpha- $2 \mathrm{~b}$ and oral tegafur/uracil with or without intravenous cisplatin was considered effective, while the efficacy of the combination therapy of interferon-alpha- $2 b$ and intravenous 5-fluorouracil was uncertain.

It has been controversial whether oral administration of tegafur/uracil improves the prognosis of patients with advanced unresectable hepatocellular carcinoma $(9,10)$. However, recently, the effectiveness of combination chemotherapy using oral tegafur/uracil has been reported $(11,12)$. 

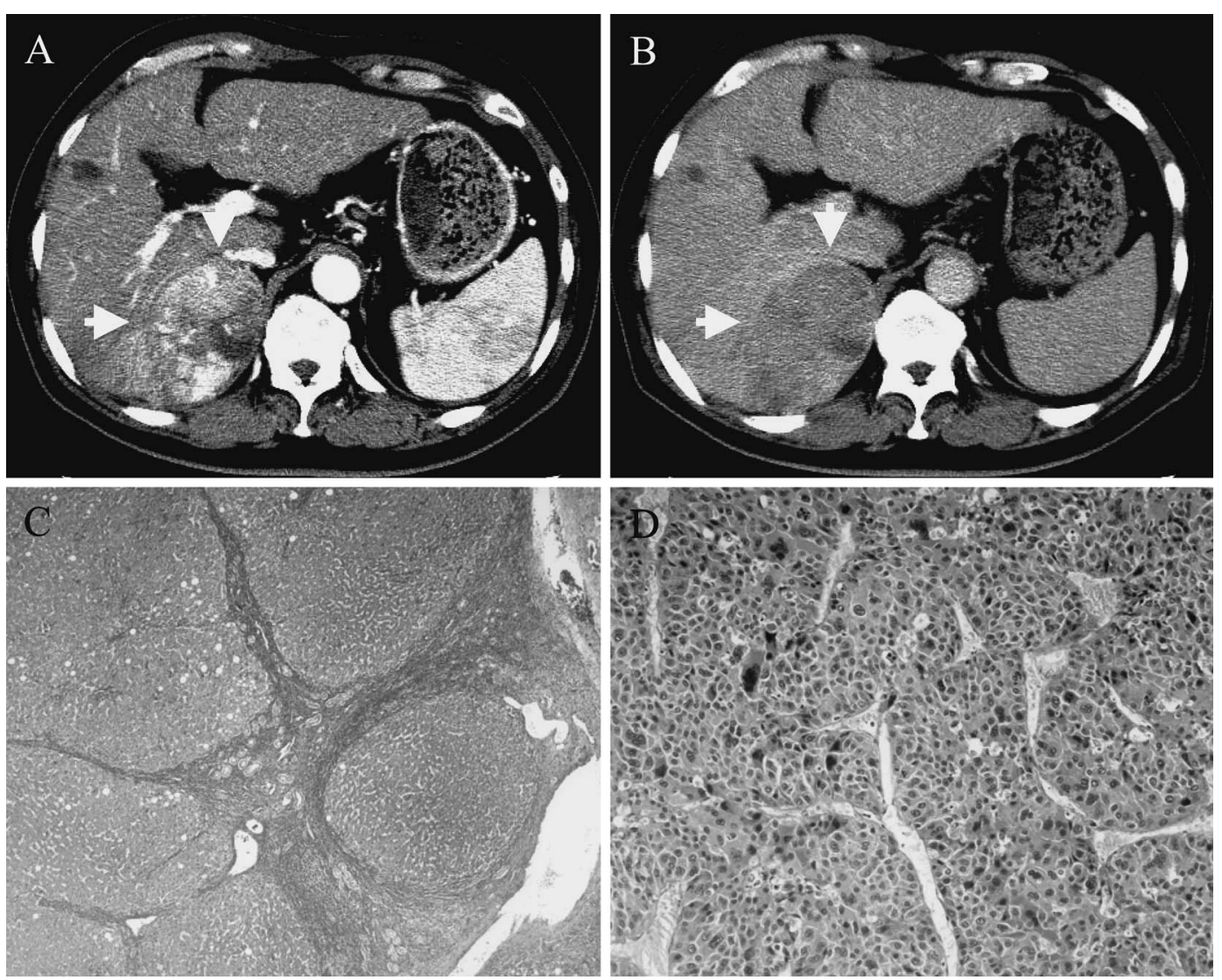

Figure 1. Demonstration of hepatocellular carcinoma by enhanced computed tomography and histological features of the non-tumorous lesion and tumorous lesion. A: Enhanced computed tomography shows a hepatic mass with early-phase hyperattenuation, measuring $8.5 \times 5.4 \mathrm{~cm}$ in the posterior segment of the liver (arrow). B: The hepatic mass shows late-phase hypoattenuation (arrow). C: Histological features of the resected non-tumorous lesion shows cirrhosis (HE stain, $\times 25$ ). D: Histological features of the resected tumorous lesion shows a moderately differentiated hepatocellular carcinoma with an increased nuclear-cytoplasm ratio and nuclear atypia, which proliferated in a trabecular pattern $(\mathrm{HE}, \times \mathbf{1 0 0})$.

Tegafur/uracil consists of tegafur and uracil in a $1: 4$ molar ratio. Tegafur is metabolized to 5-fluorouracil in the liver, and uracil potentiates the efficacy of tegafur by inhibiting dihydropyrimidine dehydrogenase activity. Hepatocellular carcinoma is reported to have a relatively high dihydropyrimidine dehydrogenase activity (13). Furthermore, oral tegafur/uracil is facile. Thus, the administration of tegafur/ uracil to patients with hepatocellular carcinoma may be reasonable.

In a recent report from Japan, major extrahepatic metastasis sites are confirmed to be lung, bone, and lymph node (3). Peritoneal dissemination is rare and usually is a consequence of rupture of the primary tumor $(3,14)$. Furthermore, peritoneal dissemination is reported to be a complication of hepatectomy, microwave coagulation therapy, ethanol injection therapy, and percutaneous needle biopsy (15-21). In the present case, there was no history of rupture of the primary tumor, and the interval from hepatectomy to the detection of peritoneal dissemination was 3 months. We speculate that, in our case, peritoneal dissemination might have been the result of intraoperative contamination. On the other hand, surgical treatment is recommended for peritoneal dissemination of hepatocellular carcinoma, based on the resistance of extrahepatic metastasis to systemic chemotherapy (16). However, we consider that outcomes of resection for peritoneal dissemination may depend on the state of tumors and the skills of surgeons.

The present patient developed general fatigue and nausea during the chemotherapy using intravenous 5-fluorouracil or cisplatin. On the other hand, during the combination therapy of interferon-alpha- $2 b$ and oral tegafur/uracil, no severe adverse events occurred, and white blood cell and neutrophil counts were maintained at $\geq 3000 / \mathrm{mm}^{3}$ and $\geq 1500 / \mathrm{mm}^{3}$, respectively. He died of necrotizing cellulites; however, the association between necrotizing cellulites and the chemotherapy was uncertain.

In conclusion, a combination therapy of interferon-alpha$2 \mathrm{~b}$ and oral tegafur/uracil is facile and may be effective for extrahepatic metastasis of hepatocellular carcinoma. However, the efficacy of oral tegafur/uracil for patients with advanced unresectable hepatocellular carcinoma has been controversial. On the other hand, the efficacy of a combination 


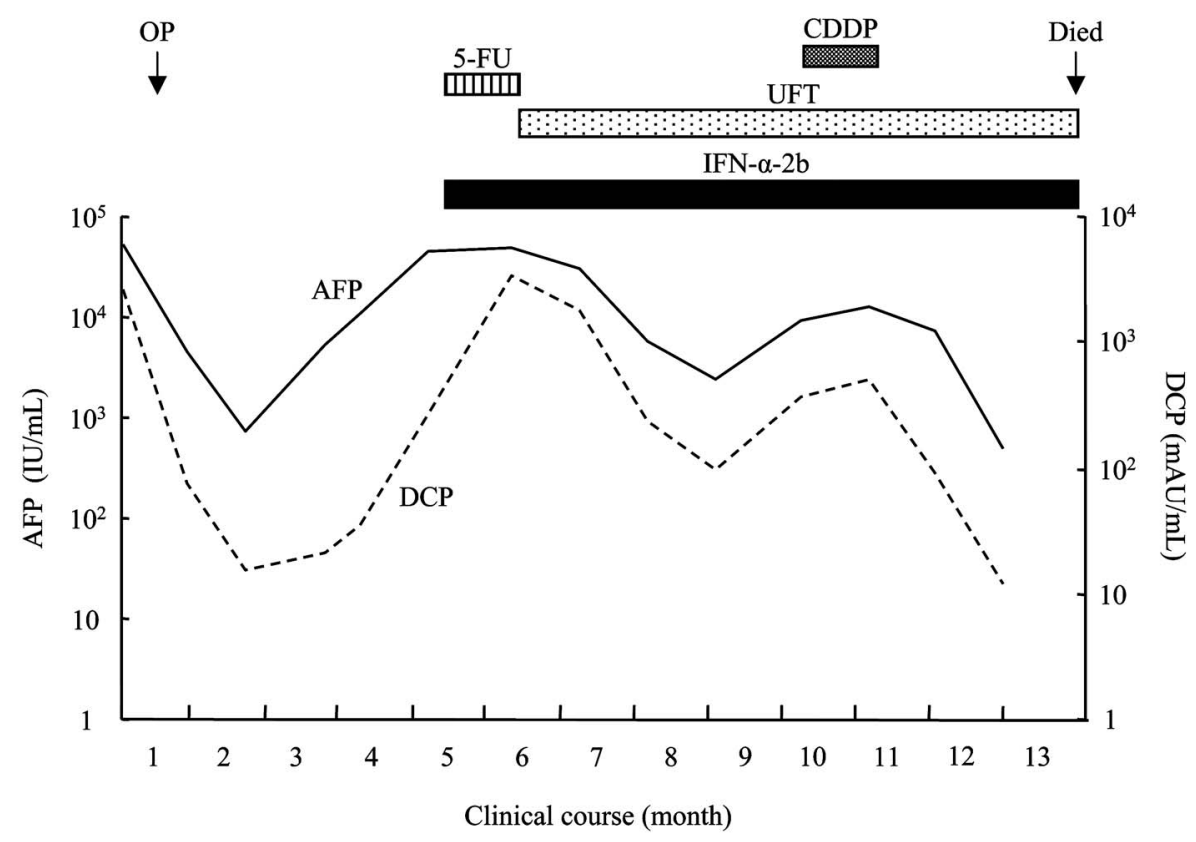

Figure 2. Patient's clinical course. OP, operation; AFP, alpha-fetoprotein; DCP, des-gamma-carboxy prothrombin, IFN- $\alpha-2 b$, interferon-alpha-2b; 5-FU, 5-fluorouracil; UFT, tegafur/uracil; CDDP, cisplatin.
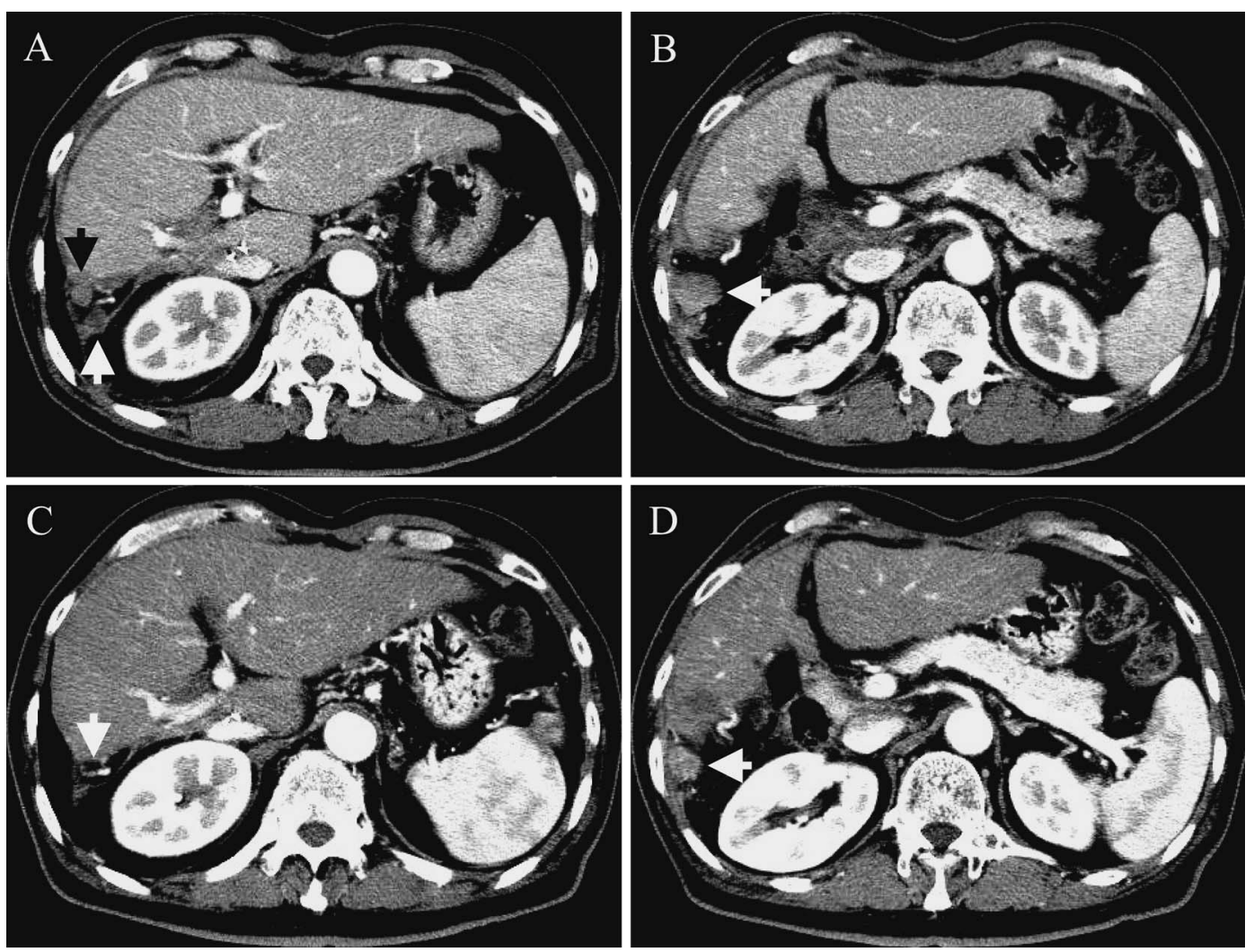

Figure 3. Demonstration of hepatocellular carcinoma disseminated to the peritoneum by computed tomography before and after the chemotherapy. A: Computed tomography shows the second (white arrow) and the third (black arrow) largest lesions of peritoneal dissemination before the chemotherapy, measuring $1.1 \times 1.0 \mathrm{~cm}$ and $1.0 \times 0.8 \mathrm{~cm}$, respectively. B: Computed tomography shows the largest lesion of peritoneal dissemination before the chemotherapy, measuring $2.6 \times$ $2.1 \mathrm{~cm}$ (arrow). C: Computed tomography shows complete disappearance of disseminated lesions after the chemotherapy (arrow). D: Computed tomography shows a reduced disseminated lesion after the chemotherapy, measuring $1.6 \times 1.3 \mathrm{~cm}$ (arrow). 
therapy of interferon-alpha and intra-arterial 5-fluorouracil for patients with major portal vein tumor thrombosis has been reported. We consider that, in order to compare the efficacy of a combination therapy of interferon-alpha and oral tegafur/uracil to that of interferon-alpha and intravenous 5fluorouracil, a prospective study for patients who develop extrahepatic metastasis of hepatocellular carcinoma is warranted.

\section{References}

1. Bosch FX, Ribes J, Diaz M, Cleries R. Primary liver cancer: worldwide incidence and trends. Gastroenterology 127: S5-S16, 2004

2. Llovet JM, Burroughs A, Bruix J. Hepatocellular carcinoma. Lancet 362: 1907-1917, 2003.

3. Ikai I, Arii S, Ichida T, et al. Report of the 16th follow-up survey of primary liver cancer. Hepatol Res 32: 163-172, 2005.

4. Ikai I, Arii S, Kojiro M, et al. Reevaluation of prognostic factors for survival after liver resection in patients with hepatocellular carcinoma in a Japanese nationwide survey. Cancer 101: 796-802, 2004

5. Kaneko S, Urabe T, Kobayashi K. Combination chemotherapy for advanced hepatocellular carcinoma complicated by major portal vein thrombosis. Oncology 62: S69-S73, 2002.

6. Ota H, Nagano H, Sakon M, et al. Treatment of hepatocellular carcinoma with major portal vein thrombosis by combined therapy with subcutaneous interferon-alpha and intra-arterial 5fluorouracil; role of type 1 interferon receptor expression. $\mathrm{Br} \mathrm{J}$ Cancer 93: 557-564, 2005.

7. Chung YH, Song IH, Song BC, et al. Combined therapy consisting of intraarterial cisplatin infusion and systemic interferon-alpha for hepatocellular carcinoma patients with major portal vein thrombosis or distant metastasis. Cancer 88: 1986-1991, 2000.

8. Miyamoto A, Umeshita K, Sakon M, et al. Advanced hepatocellular carcinoma with distant metastases, successfully treated by a combination therapy of alpha-interferon and oral tegafur/uracil. J Gastroenterol Hepatol 15: 1447-1451, 2000.

9. Ikeda K, Saitoh S, Koida I, et al. A prospective randomized evaluation of a compound of tegafur and uracil as an adjuvant chemotherapy for hepatocellular carcinoma treated with transcatheter arterial chemoembolization. Am J Clin Oncol 18: 204-210, 1995.

10. Ishikawa $T$, Ichida $T$, Sugitani $S$, et al. Improved survival with oral administration of enteric-coated tegafur/uracil for advanced stage IV-A hepatocellular carcinoma. J Gastroenterol Hepatol 16: 452-459, 2001

11. Maeda $T$, Itasaka $H$, Takenaka $K$, et al. Low-dose cisplatin plus oral tegafur and uracil for the treatment of lung metastases of he- patocellular carcinoma. Hepatogastroenterology 50: 1583-1586, 2003.

12. Seki S, Yamada T, Kawakita N, Masuichi H, Kitada T, Sakaguchi H. A new chemotherapeutic regimen for advanced unresectable hepatocellular carcinoma. Hepatogastroenterology 50: 1598-1602, 2003.

13. Jiang W, Lu Z, He Y, Diasio RB. Dihydropyrimidine dehydrogenase activity in hepatocellular carcinoma: implication in 5fluorouracil-based chemotherapy. Clin Cancer Res 3: 395-399, 1997.

14. Sonoda T, Kanematsu T, Takenaka K, Sugimachi K. Ruptured hepatocellular carcinoma evokes risk of implanted metastases. J Surg Oncol 41: 183-186, 1989.

15. Eriguchi N, Aoyagi S, Okuda K, et al. Successful surgical treatment for implanted intraperitoneal metastases of hepatocellular carcinoma. J Hepatobiliary Pancreat Surg 7: 520-523, 2000.

16. Nakayama H, Takayama $T$, Makuuchi $M$, et al. Resection of peritoneal metastases from hepatocellular carcinoma. Hepatogastroenterology 46: 1049-1052, 1999.

17. Yoshida H, Onda M, Tajiri $T$, et al. Successful surgical treatment of peritoneal dissemination of hepatocellular carcinoma. Hepatogastroenterology 49: 1663-1665, 2002.

18. Sato M, Tokui K, Watanabe Y, et al. Generalized intraperitoneal seeding of hepatocellular carcinoma after microwave coagulation therapy: a case report. Hepatogastroenterology 46: 2561-2564, 1999.

19. Miki K, Makuuchi M, Takayama T, et al. Peritoneal seeding of hepatocellular carcinoma after ethanol injection therapy. Hepatogastroenterology 47: 1428-1430, 2000.

20. Takahashi H, Konishi M, Nakagohri T, et al. Aggressive multimodal treatment for peritoneal dissemination and needle tract implantation of hepatocellular carcinoma: a case report. Jpn J Clin Oncol 34: 551-555, 2004

21. Uenishi T, Kubo S, Hirohashi K, et al. Successful surgical control for hepatocellular carcinoma disseminated to the peritoneum: a case report. Hepatogastroenterology 49: 532-534, 2002.

(C) 2007 The Japanese Society of Internal Medicine http://www.naika.or.jp/imindex.html 